\title{
Methicillin-Resistant Staphylococci: Genetics of the Minority Population
}

\author{
By S. J. SELIGMAN \\ University of California, Centre for the Health Sciences, \\ Los Angeles, California, U.S.A.
}

(Received 1 September 1965)

\begin{abstract}
SUMMARY
A methicillin-resistant strain of Staphylococcus aureus (no. 5982) contained cocci with differing intrinsic resistances to methicillin. Most of the population was slightly more resistant than a typical methicillin-sensitive staphylococcal isolate, but a few were mutants with ability to grow at markedly increased concentrations of methicillin $(500 \mu \mathrm{g} . / \mathrm{ml}$. or more). Penicillinase production was not essential for methicillin resistance since a penicillinase-negative variant of strain $\mathbf{5 9 8 2}$ was able to produce penicillinase-negative strains with high degrees of methicillin resistance. Methicillin-sensitive strains also gave rise to mutants with increased intrinsic resistance to methicillin, but, in the mutants from methicillin-resistant strains, the increase in resistance was much greater. The mutants were characteristically slower growing than the wild type. Many were small colony variants which reverted to the parent type when passed in liquid media. With $5 \% \mathrm{NaCl}$-methicillin plates strain 5982 exhibited a phenotypic increase in resistance. On such plates a few L-type colonies were produced at high concentrations of methicillin.
\end{abstract}

\section{INTRODUCTION}

Methicillin is effective against penicillinase-producing staphylococci because of its relative resistance to penicillin- $\beta$-lactamase, the staphylococcal enzyme which inactivates benzylpenicillin. Although at high concentrations methicillin is inactivated at a rate about one-thirtieth that of benzylpenicillin, the extremely poor affinity of the enzyme for methicillin (Novick, 1962; Richmond, 1963) probably results in negligible inactivation under clinical conditions. A few naturally occurring methicillin-resistant staphylococci have been isolated, as first reported by Jevons (1961) and on occasion have given rise to severe illness (Stewart \& Holt, 1963). Such isolates have been reported to be penicillinase producers; hence they were capable of some degree of methicillin inactivation. However, the findings of Sutherland \& Rolinson (1964) indicated that methicillin-resistant staphylococci, which had been isolated by several laboratories in England and in Europe, consisted of a majority population with a comparatively slight increase in methicillin resistance, and a minority population with a much greater increase in resistance. In the work described here the characteristics of the minority population were further investigated and compared with methicillin-sensitive strains. 


\title{
METHODS
}

Media. Trypticase soy agar plates (TSA, Baltimore Biological Laboratories) containing methicillin were prepared by adding various amounts of methicillin to liquid TS agar cooled to $45^{\circ}$. Liquid cultures were made in trypticase soy (TS) broth.

Organisms. The methicillin-resistant Staphylococcus aureus (no. 5982), was originally isolated by Eriksen \& Erichsen (1963) and was kindly supplied by Drs Kagan and Martin. The methicillin-sensitive strains were the penicillin-sensitive Oxford staphylococcus (209P) and a penicillin-resistant clinical isolate, no."97990. Stock cultures were kept by monthly passage of an isolated colony on TS agar. Suspected mutants were streaked on TS agar from isolated colonies for at least three successive transfers before testing.

Inoculation of plates. Overnight broth cultures were diluted in TS broth and streaked linearly in $0.05 \mathrm{ml}$. amounts on methicillin plates, 4 dilutions/plate. The plates were read daily for 2 days. When hypertonic media were used or when small colony variants were being studied, incubation was continued for 6 days. The methicillin-resistance of the small colony variants was studied by fishing an isolated colony into $2 \mathrm{ml}$. of TS broth which was considered as the undiluted inoculum.

\section{RESULTS}

The minority population consisted of organisms which grew at concentrations of methicillin which inhibited the majority (Table 1). After incubation for 1 day only a few of the minority population were visible on methicillin plates, but by 2 days the number of colonies was of the same order as that obtained after more prolonged

Table 1. Colony counts of a methicillin-sensitive strain of Staphylococcus aureus, no. 5982, on methicillin plates

\begin{abstract}
Dilutions of an overnight culture were inoculated in $0.05 \mathrm{ml}$. amounts on methicillin TS agar plates and incubated for 2 days. $+=>100$ but discrete, $++=$ too numerous to count. $+++=$ confluent growth.
\end{abstract}

Dilution of culture

$\begin{array}{crrrr}\begin{array}{c}\text { Methicillin } \\ (\mu \mathrm{g} . / \mathrm{ml} .)\end{array} & \overbrace{\mathbf{1 0}}^{\mathbf{6}} & \mathbf{1 0}^{-\mathbf{4}} & \begin{array}{r}\mathbf{1 0}^{-2} \\ \text { colony count }\end{array} & \text { Undiluted } \\ \mathbf{0} & \mathbf{6 2} & ++ & +++ & +++ \\ \mathbf{5} & \mathbf{6 0} & ++ & +++ & +++ \\ \mathbf{1 2 . 5} & \mathbf{0} & \mathbf{3} & + & +++ \\ \mathbf{2 5} & \mathbf{0} & 1 & + & +++ \\ \mathbf{5 0} & \mathbf{0} & \mathbf{1} & \mathbf{3 9} & +++ \\ \mathbf{1 2 5} & \mathbf{0} & \mathbf{0} & \mathbf{1 5} & ++ \\ \mathbf{2 5 0} & \mathbf{0} & \mathbf{0} & \mathbf{9} & + \\ \mathbf{5 0 0} & \mathbf{0} & \mathbf{0} & \mathbf{2} & +\end{array}$

incubation. Except for an occasional small colony isolated from a barely inhibitory concentration, colonies isolated from the minority population proved to be mutants with increased intrinsic resistance to methicillin. In colonial appearance the parent strain was a typical Staphylococcus aureus. The mutants, however, varied in colour from off-white to a deeply pigmented yellow-orange when plated either on methicillin agar or on TS agar. 
Considerable variation in the number of colonies isolated on the lower concentrations of methicillin was found in successive experiments. At methicillin $5 \mu \mathrm{g} . / \mathrm{ml}$. the results were especially variable. In some experiments the number of colonies was the same as on TS agar while in others there were $10^{4}$-fold decreases in count. Hence, it is not possible to give a precise estimate of the number of mutants from the data in Table 1. Experiments done with ten-fold bacterial dilutions indicated that the colony count was not always proportional to the dilution factor. Inoculation of several plates at the same dilution indicated that the number of plates incubated in a stack, the place of the stack in the incubator, and the number of plates in the incubator influenced the colony count. Similar variability in quantitative results was found with Escherichia coli and ampicillin by Seligman \& Hewitt (1965).

Isolates from barely inhibitory concentrations of methicillin exhibited degrees of increased resistance, varying from $12.5 \mu \mathrm{g} . / \mathrm{ml}$. to greater than $500 \mu \mathrm{g} . / \mathrm{ml}$. In Table 2 the resistance pattern of a mutant (no. 5982/M 10) isolated from a $500 \mu \mathrm{g} . / \mathrm{ml}$., plate illustrates that the number of colony-forming particles able to grow on $500 \mu \mathrm{g}$. plates was substantially greater than with the parent strain. A spontaneously occurring back mutant (no. 5982/M 10/399) was of particular interest, since it grew as a confluent streak on a $500 \mu \mathrm{g}$. plate when an undiluted overnight culture was inoculated, but the majority of its population was inhibited by 12.5 to $25 \mu \mathrm{g}$.; and at 50 to $500 \mu \mathrm{g}$. only a few colonies were produced at a $10^{-2}$ dilution (Table 2).

Table 2. Minority populations mutants plated on methicillin plates

Strain 5982/M 10 was isolated from a methicillin $500 \mu \mathrm{g} . / \mathrm{ml}$. plate. Strain 5982/M 10/399 was isolated from 5982/M 10 on TS agar. Method and terminology as in Table 1.

\begin{tabular}{|c|c|c|c|c|c|c|c|c|}
\hline \multirow{3}{*}{$\begin{array}{l}\text { Methicillin } \\
(\mu \mathrm{g} \cdot / \mathrm{ml} .)\end{array}$} & \multicolumn{4}{|c|}{$5982 / \mathrm{M} 10$} & \multicolumn{4}{|c|}{$5982 /$ M $10 / 399$} \\
\hline & \multicolumn{4}{|c|}{ Dilution } & \multicolumn{4}{|c|}{ Dilution } \\
\hline & $10^{-6}$ & $10^{-4}$ & $10^{-2}$ & $\begin{array}{r}\text { Undiluted } \\
\text { Colo }\end{array}$ & $\begin{array}{l}10^{-6} \\
\text { ies }\end{array}$ & $10^{-4}$ & $10^{-2}$ & Undiluted \\
\hline 0 & 39 & ++ & +++ & +++ & 34 & ++ & +++ & $+t+$ \\
\hline $\mathbf{5}$ & 38 & ++ & +++ & +++ & 24 & ++ & +++ & +++ \\
\hline $12 \cdot 5$ & $\mathbf{3 7}$ & ++ & $++t$ & $++t$ & $\mathbf{0}$ & 19 & +++ & +++ \\
\hline 25 & 37 & ++ & +++ & $++t$ & $\mathbf{0}$ & 1 & +++ & +++ \\
\hline $\mathbf{5 0}$ & 12 & ++ & +++ & +++ & $\mathbf{0}$ & 0 & 18 & +++ \\
\hline 125 & 12 & ++ & +++ & $+t+$ & 0 & $\mathbf{0}$ & 7 & +++ \\
\hline 250 & 17 & ++ & $++t$ & +++ & 0 & $\mathbf{0}$ & 5 & $+t+$ \\
\hline 500 & 10 & ++ & +++ & $++t$ & $\mathbf{0}$ & 0 & $\mathbf{0}$ & $+t+$ \\
\hline
\end{tabular}

Such a marked inoculum effect with confluent growth of a concentrated inoculum is characteristic of penicillinase activity; however, significant inactivation of methicillin could not be demonstrated by iodometric titration (Perret, 1954) or by a modified Gots test (Gots, 1945).

After numerous serial transfers of isolated colonies on TS agar strain 5982 lost its ability to form penicillinase as judged by a negative Gots test, a negative Novick test (Novick, 1963; Novick \& Richmond, 1965), and lack of ability to grow as a confluent streak of concentrated inocula on concentrations of penicillin 
which were inhibitory to the majority. Comparison with a penicillinase-positive variant of an earlier passage revealed that both strains had a similar pattern of methicillin resistance, except that growth of the penicillinase-negative variant was frequently less on the $5 \mu \mathrm{g}$. plates. Isolates of the penicillinase-negative variant from $500 \mu \mathrm{g}$. plates proved to be mutants with large increases in methicillin resistance, comparable to their penicillinase-producing counterparts. Both the penicillinase-positive and -negative variants were resistant to streptomycin and tetracycline but were sensitive to erythromycin, kanamycin or chloramphenicol. Additional studies on a series of such penicillinase-negative, methicillin-resistant mutants will be reported elsewhere.

Four methicillin-resistant penicillinase-positive mutants were tested after serial passage in antibiotic-free media. The three highly resistant strains reverted to a population with a resistance pattern similar to that of the parent strain. The fourth, in which the increased resistance was of relatively low degree, maintained its resistance after fourteen serial passages. (Seligman, 1966)

Table 3. Methicillin-sensitive staphylococci on methicillin plates

Strain $209 \mathrm{P}$ is penicillinase-negative. Strain 97990 is pencillinase-positive. Method and terminology as in Table 1.

\begin{tabular}{|c|c|c|c|c|c|c|c|c|}
\hline \multirow{4}{*}{$\begin{array}{r}\text { Methicillin } \\
\text { ( } \mu \mathrm{g} . / \mathrm{ml} .)\end{array}$} & \multicolumn{4}{|c|}{$209 \mathrm{P}$} & \multicolumn{4}{|c|}{97990} \\
\hline & \multicolumn{4}{|c|}{ Dilution } & \multicolumn{4}{|c|}{ Dilution } \\
\hline & $10^{-6}$ & $10^{-4}$ & $10^{-2}$ & Undiluted & $10^{-6}$ & $10^{-4}$ & $10^{-2}$ & Undiluted \\
\hline & \multicolumn{8}{|c|}{ Colonies } \\
\hline 0 & 84 & ++ & +++ & $++t$ & 61 & ++ & +++ & $+t+$ \\
\hline 1 & 1 & 2 & $+t$ & +++ & 48 & ++ & +++ & $+t+$ \\
\hline 2 & 0 & 0 & 50 & ++ & 17 & ++ & +++ & $+t+$ \\
\hline 3 & o & 0 & 13 & ++ & 0 & 0 & 9 & $+t$ \\
\hline 4 & o & $\mathbf{0}$ & $\mathbf{5}$ & ++ & $\mathbf{0}$ & 0 & 0 & 14 \\
\hline $\mathbf{5}$ & 0 & 0 & 0 & 0 & 0 & 0 & 0 & 1 \\
\hline
\end{tabular}

Step-wise resistance

Mutations to increased resistance to the penicillins usually consist of relatively small increments in step-wise resistance in both Gram-positive (Demerec, 1945; Hotchkiss, 1951) and Gram-negative organisms (Roantree \& Steward, 1965; Seligman \& Hewitt, 1965; Banič, 1959). However, the relative increment in resistance from $5 \mu \mathrm{g} . / \mathrm{ml}$. to greater than $500 \mu \mathrm{g} . / \mathrm{ml}$. in many of the methicillin-resistant mutants was much greater than that found with mutations to the other penicillins. Consequently, such mutations to methicillin resistance resembled the one-step mutations to high degrees of resistance which are characteristic of many streptomycin-resistant mutants. In contrast, the penicillinase-negative Oxford staphylococcus (209P) and a typical penicillinase-producing clinical isolate contained majority populations which were inhibited by methicillin 2 and $3 \mu \mathrm{g} . / \mathrm{ml}$., respectively, and minority populations almost completely inhibited by methicillin $5 \mu \mathrm{g} . / \mathrm{ml}$. (Table 3). These strains also produced mutants which, after at least 3 and 5 steps respectively, consisted predominantly of populations resistant to methicillin 25 and $10 \mu \mathrm{g} . / \mathrm{ml}$. 


\section{Small colony variants}

Small colonies appeared on methicillin plates after incubation for 1 to 4 days but subculture and further observation revealed that they had diverse genetic backgrounds. Some, particularly in crowded areas at borderline inhibitory concentrations, proved to be phenotypic variants of the parent strain. Others grew to larger size on more prolonged incubation and were mutants with increased methicillin resistance. On TS agar they had a slower growth rate than the parent strain. In the third group, especially those which first became visible after incubation for 3-4 days on methicillin plates, the final size of a colony on TS agar was distinctly smaller than the parent strain. These small colony mutants are comparable to the small colony variants described after exposure to benzylpenicillin (Youmans, Williston \& Simon, 1945). On TS agar their colonies were regular and the morphology by Gram stain was normal. Subinhibitory concentrations of methicillin were sometimes associated with abnormally swollen, variably sized, cocci (Barber, 1964).

The small colony variants described here were almost always more resistant to methicillin than was the parent strain. Some grew in undiminished numbers in methicillin up to $500 \mu \mathrm{g} . / \mathrm{ml}$., the highest concentrations at which most strains were tested. In the more heavily streaked areas of TS agar plates, larger colonies were seen occasionally and when isolated usually proved to be back mutants giving colonies larger than their small-colony variant (Fig. 1). Continued incubation resulted in an increase in the number of larger colonies, particularly in the less heavily inoculated areas; this finding is attributable in part to intracolonial mutation (Fig. 2). Frequently, the back mutants had also regained the pigmentation and methicillin sensitivity of the parent strain, but some retained the altered pigmentation and sometimes the approximate methicillin resistance of their small colony variant. Small colony variants have been maintained by passage of isolated colonies on TS agar for more than 25 consecutive streakings. When grown in broth, however, the faster-growing back mutants became the predominant organisms. Such overgrowth was sometimes significant after one transfer and, together with phenotypic variation and intracolonial mutation, accounts for previous difficulties in estimating antibiotic resistance in the small colony variants (Youmans et al. 1945).

\section{Resistance in hypertonic media and L-type growth}

Growth in media containing $5 \%(\mathrm{w} / \mathrm{v}) \mathrm{NaCl}$ has been reported to increase the resistance of methicillin-resistant staphylococci (Barber, 1964). In the present work experiments with methicillin plates containing $5 \%(\mathrm{w} / \mathrm{v}) \mathrm{NaCl}$ usually revealed growth of a majority of the parent population on methicillin $50 \mu \mathrm{g} . / \mathrm{ml}$. plates, roughly a tenfold increase. Isolates from such plates did not exhibit increased methicillin resistance, thereby confirming the phenotypic nature of increased resistance of the majority population on hypertonic media. By contrast, growth of the minority population was inhibited on hypertonic methicillin plates. Small numbers of $\mathrm{L}$-type colonies were isolated on methicillin $+\mathrm{NaCl}+\mathrm{TS}$ agar plates, especially at methicillin 500-1000 $\mu \mathrm{g}$. $/ \mathrm{ml}$. Such colonies were similar to the ones previously described from methicillin-resistant staphylococci (Kagan, Martin \& Stewart, 1964) and could be propagated on methicillin $+\mathrm{NaCl}+\mathrm{TS}$ agar plates. Added animal serum was not essential for the isolation or propagation of L-type growth. 


\section{DISCUSSION}

The mechanism of methicillin resistance has been a controversial subject. Several workers have shown that methicillin-resistant strains can inactivate methicillin to a greater degree than can methicillin-sensitive strains (Eriksen \& Erichsen, 1964b; Gravenkemper, Brodie \& Kirby, 1965). However, in these experiments the concentrations of methicillin were usually such that greater growth of the methicillinresistant strains was permitted. Disproportionate increase in penicillinase production might account for increased inactivation of methicillin. Under appropriate conditions (Ayliffe \& Barber, 1963; Knox \& Smith, 1963), comparable amounts of methicillin were destroyed by both methicillin-sensitive and methicillin-resistant strains. Furthermore, it is clear that the degree of inactivation of methicillin could not account for the increase in methicillin resistance observed in the highly resistant mutants. The penicillinase-negative variant which maintained the ability to produce highly resistant mutants indicates that penicillinase production did not contribute significantly to such methicillin resistance. Eriksen \& Erichsen (1964a), however, encountered a penicillinase-negative variant of a methicillin-resistant strain which, in their studies, showed a decrease in methicillin resistance. Penicillinase-negative variants in methicillin-sensitive strains, and presumably in the current methicillinresistant strain, result from the loss of an extra-chromosomal element or plasmid (Novick, 1963).

A characteristic of significant penicillinase production is the ability of surface colonies from heavy inocula to grow as confluent streaks at concentrations of penicillin considerably greater than that which inhibited the production of isolated colonies. As already mentioned, a mutant was isolated which showed such an inoculum effect on methicillin plates but in which a significant degree of methicillin inactivation was not found. The explanation of such an inoculum effect remains conjectural; it might result from intercellular utilization of enzymes involved with cell wall synthesis. Barber (1964) observed results consistent with an inoculum effect in methicillin-resistant staphylococci. However, our studies, in confirmation of those of Sutherland \& Rolinson (1964), indicate that most resistant mutants are able to grow as isolated colonies on methicillin plates. Although it is difficult to give a precise estimate of mutant frequency (see above), in both methicillin-sensitive and methicillin-resistant strains, approximately one organism in $10^{4}$ is a mutant. With similar techniques the order of magnitude of the frequency of resistant mutants of Escherichia coli to ampicillin (Seligman \& Hewitt, 1965) was found to be similar to that found in the present work for methicillin resistance in staphylococci. Therefore, the methicillin-resistant staphylococci do not appear to be characterized by an excessive rate of mutation for this marker. The majority of organisms in a population of the methicillin-resistant strain is more resistant than the majority in the sensitive strain, but this difference is relatively slight. Accordingly, the distinguishing property of the resistant strain is its capacity to mutate to greatly increased methicillin resistance.

Step-wise increase in resistance to penicillin was found by Banič (1959) to be polygenic. It has not been determined whether more than one locus is capable of mutating to the currently described large-step increase in methicillin resistance. The alterations in biosynthetic processes present in the naturally-occurring resistant 
strains which are associated with the modest increase in resistance may be those which permit mutation to greatly increased methicillin resistance. Study of such altered metabolic pathways may reveal additional evidence of the biochemical defect resulting from the action of penicillin.

The plethora of variants with diminished colonial size indicates that the metabolic pathways associated with methicillin resistance usually result in a slower growth rate in antibiotic-free media. The term G colony (Hadley, Delves \& Klimek, 1931), sometimes used in association with small colony variants, does not seem appropriate since it was originally used to describe small colony variants thought to reproduce by gonidia.

This work was supported in part by United States Public Health Service Grant no. HD 00303, awarded to Dr W. L. Hewitt, and was done during the tenure of a United States Public Health Service Research Career Development Award. Methicillin was kindly supplied by Bristol Laboratories, Syracuse, New York. The technical assistance of Miss Britt Westerdahl is gratefully acknowledged.

\section{REFERENCES}

Ayliffe, G. A. F. \& Barber, M. (1963). Inactivation of benzyl-penicillin and methicillin by hospital staphylococci. Br. med. J. ii, 202.

BANIČ, S. (1959). Transduction to penicillin and chloramphenicol resistance in Salmonella typhimurium. Genetics, 44, 449.

Barber, M. (1964). Naturally occurring methicillin-resistant staphylococci. J. gen. Microbiol. 35, 183.

Demerec, M. (1945). Production of staphylococcus strains resistant to various concentrations of penicillin. Proc. natn Acad. Sci., U.S.A. 31, 16.

Eriksen, K. R. \& Erichsen, I. (1963). Resistance to the newer penicillins. Br. med. J. i, 746.

ErIKsen, K. R. \& Erichsen, I. (1964a). Resistance to methicillin, isoxazolyl penicillins and cephalothin in Staphylococcus aureus. Acta. path. microbiol. scand. 62, 255.

ErIKSEN, K. R. \& ErichSEN, I. (1964b). Inactivation of methicillin, oxacillin, cloxacillin, and cephalothin by staphylococcal penicillinase. Acta path. microbiol. scand. 62, 399 .

Gots, J. S. (1945). The detection of penicillinase-producing properties of microorganisms. Science, 102, 309.

Gravenkemper, C. F., Brodie, J. L. \& Kirby, W. M. M. (1965). Resistance of coagulasepositive staphylococci to methicillin and oxacillin. J. Bact. 89, 1005.

Hadley, P., Delves, E. \& Klimek, J. (1931). The filtrable forms of bacteria. I. A filtrable stage in the life history of the Shiga dysentery bacillus. J. infect. Dis. 48, 1.

HотсHкIss, R. D. (1951). Transfer of penicillin resistance in pneumococci by the desoxyribonucleate derived from resistant cultures. Cold Spr. Harb. Symp. quant. Biol. 16, 457.

Jevons, M. P. (1961). 'Celbenin'-resistant staphylococci. Br. med. J. i, 124.

Kagan, B. M., Martin, E. R. \& Stewart, G. T. (1964). L-form induction of naturally occurring methicillin-resistant strains of Staphylococcus aureus. Nature, Lond. 203, 1031.

KNox, R. \& SMrTH, J. T. (1963). Stability of methicillin and cloxacillin to staphylococcal penicillinase. $B r$. med. $J$. ii, 205.

Novick, R. P. (1962). Staphylococcal penicillinase and the new penicillins. Biochem. J. 83, 229.

Novick, R.P. (1963). Analysis by transduction of mutations affecting penicillinase formation in Staphylococcus aureus. J. gen. Microbiol. 33, 121.

Novick, R. P. \& Richmond, M. H. (1965). Nature and interactions of the genetic elements governing penicillinase synthesis in Staphylococcus aureus. J. Bact. 90, 467.

Pernett, C. J. (1954). Iodometric assay of penicillinase. Nature, Lond. 174, 1012. 
Richmond, M. H. (1963). Purification and properties of the exopenicillinase from Staphylococcus aureus. Biochem. J. 88, 452.

Roantree, R. J. \& Steward, J.P. (1965). Mutations to penicillin resistance in the Enterobacteriaceae that affect sensitivity to serum and virulence for the mouse. J. Bact. $89,630$.

Seligman, S. J. (1966). Penicillinase-negative varients of methicillin-resistant Staphyloccus aureus. Nature, Lond. (in press)

Seligman, S. J. \& Hewitr, W. L. (1965). Mutation of Escherichia coli to increased resistance to ampicillin. Antimicrobiol. Agents and Chemotherapy-1964, p. 344.

Stewart, G. T. \& Holt, R. J. (1963). Evolution of natural resistance to the newer penicillins. Br. med. J. i, 308.

Sutherland, R. \& Rolinson, G. N. (1964). Characteristics of methicillin-resistant staphylococci. J. Bact. 87, 887.

Youmans, G. P., Wichrston, E. H. \& Srmon, M. (1945). Production of small colony variants by the action of penicillin. Proc. Soc. exp. Biol. Med. 58, 56.

\section{EXPLANATION OF PLATE}

Fig. 1. Staphylococcal small-colony variants streaked on TS agar and incubated for 4 days. Larger colonies in crowded areas are back mutants with increased colonial size. In the sparser areas the disproportionately large colonies frequently contained intracolonial mutants.

Fig. 2. Staphylococcal intracolonial mutant developing in an isolated small-colony variant. The drawing illustrates the change which had occurred since the previous day's incubation. The smallcolony variant was distinctly more pigmented than the faster-growing back mutant. 


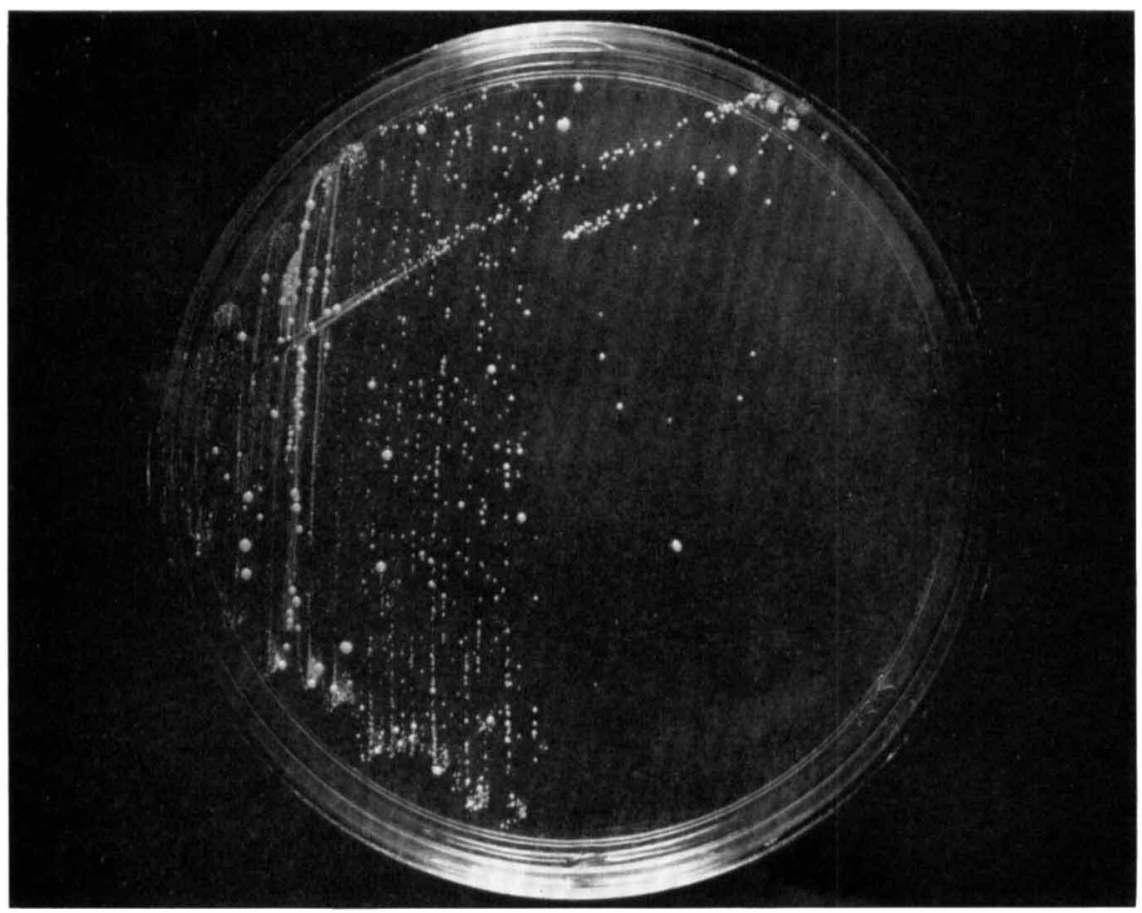

Fig. 1
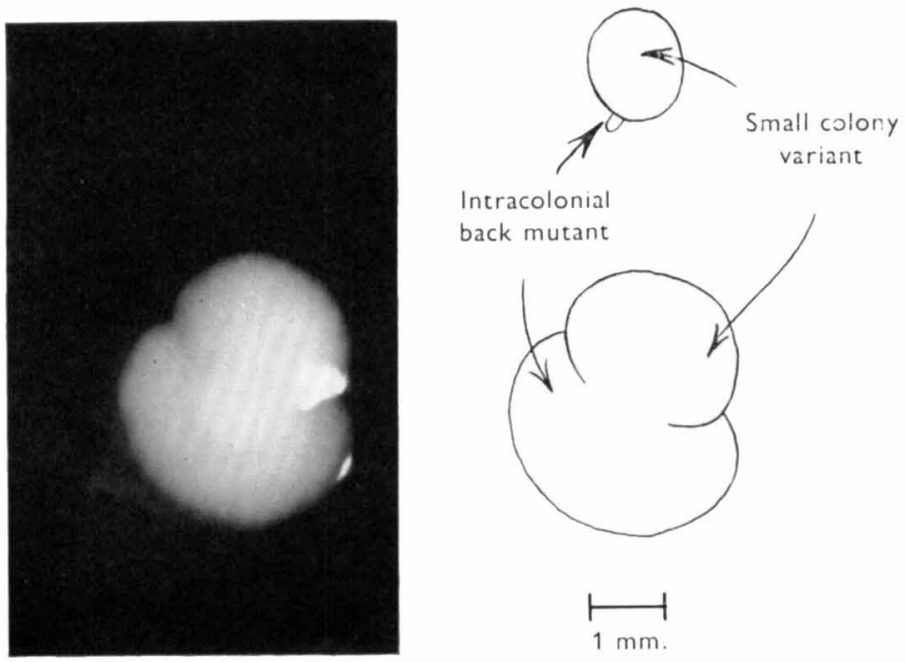

Fig. 2 